\title{
Quantum size effect induced dilute atomic layers in ultrathin Al films
}

\author{
Ying Jiang, ${ }^{1,2}$ Kehui Wu, ${ }^{1}$ Zhe Tang, ${ }^{1}$ Ph. Ebert, ${ }^{2}$ and E. G. Wang ${ }^{1}$ \\ ${ }^{1}$ Institute of Physics, Chinese Academy of Sciences, Beijing 100080, China \\ ${ }^{2}$ Institut für Festkörperforschung, Forschungszentrum Jülich GmbH, 52425 Jülich, Germany \\ (Received 11 April 2007; revised manuscript received 23 May 2007; published 10 July 2007)
}

\begin{abstract}
We illustrate using scanning tunneling microscopy and low energy electron diffraction that thin Al films grown on $\mathrm{Si}(111)-\sqrt{3} \times \sqrt{3}-\mathrm{Al}$ substrates form layers having unusual thicknesses, not compatible with a normal fcc stacking of dense $\mathrm{Al}(111)-1 \times 1$ layers (coverage $<$ wetting layer $+7 \AA$ ). This structure is shown to be based on inserted dilute $1.5 \times 1.5$ atomic layers. At a film thickness of the wetting layer $+7 \AA$, the film undergoes a phase transformation and continues to grow in the normal stacking of $\mathrm{Al}(111)-1 \times 1$ layers. The phenomenon is explained within the theory of the quantum size effects in a jellium metal combined with strain effects. We argue that the insertion of dilute atomic layers for small film thicknesses allows the $\mathrm{Al}$ film to reach thicknesses perfectly well adjusted to the minima of the oscillating electron energy, which arises from the spatial confinement of the free electrons in the thin film. In contrast, at larger thicknesses, where the electron energy differences diminish, a strain-driven phase transformation drives the system back to the classical close-packed $\mathrm{Al}(111)-1 \times 1$ fcc stacking.
\end{abstract}

DOI: 10.1103/PhysRevB.76.035409

PACS number(s): 68.55.Jk, 68.37.Ef, 73.61.At

The isotropic property of the metallic bonding drives metal ions to get as close as possible in order to maximize the overlap of the wave functions and to minimize the energy. Therefore, most metals form close-packed crystalline structures with high coordination numbers. Depending on the shape and extension of the wave functions involved, metals crystallize preferentially in the close-packed face-centered cubic or hexagonal structures or in the nearly close-packed body-centered cubic structure. In the conventional understanding, deviations from this principle should only occur if the bonds between the metal atoms become directional, i.e., exhibit covalent contributions. Here, we demonstrate, however, that a pure single element metal can also form a stack of dilute and dense close-packed atomic layers and thus deviate from the closed-packed principle in dimensionally reduced structures. The driving force is not a directional component of the metallic bond but rather a reduction of the electron energy governed by quantum size effects. Once the gain of electron energy vanishes with increasing film thickness, the whole system undergoes, however, a strain-driven phase transformation from alternating dilute and dense atomic layers to only close-packed layers.

Quantum mechanics and thus also quantum size effects (QSEs) become relevant for spatially confined structures, such as thin metal films, whose thickness is comparable to the Fermi wavelength $\lambda_{F} \cdot{ }^{1-4}$ Perhaps, the best illustrated example of the QSE are $\mathrm{Pb}$ films on $\mathrm{Si}(111)$, where various properties, such as the surface energy, the superconducting transition temperature, the interlayer spacing, and the film stability, oscillate with the film thickness. ${ }^{5-11}$ The QSE also favors magic film thicknesses, corresponding to the electron energy minima at $\lambda_{F} / 2$ intervals. ${ }^{6-8,12,13}$ This drives metal thin films with unfavored thicknesses to stabilize by relaxing the interlayer spacing. However, if the resulting increase in strain energy cannot be compensated by the gain in electron energy, metal films with such thicknesses are unstable and do not form. Al is such a material, where the mismatch between the interlayer spacing $(0.23 \mathrm{~nm})$ and the Fermi wavelength $(0.36 \mathrm{~nm})$ is too large to adjust the film thickness purely by strain, and thus bulklike close-packed Al films should be unstable for small film thicknesses. We demonstrate, however, that Al thin films grown on $\mathrm{Si}(111)-\sqrt{3} \times \sqrt{3}-\mathrm{Al}$ substrates circumvent this problem by forming a fundamentally different atomic structure consisting of dilute and dense close-packed atomic layers. This phase yields Al film thicknesses perfectly well adjusted to the minima of the QSEinduced oscillating electronic energy, something which cannot be achieved by a normal stacking of $\mathrm{Al}(111)$ layers due to the strain energy.

For our experiments, we prepared $\mathrm{Si}(111)-\sqrt{3} \times \sqrt{3}-\mathrm{Al}$ substrates by evaporating $0.15 \mathrm{ML} \mathrm{Al}$ [in terms of the atomic density of the $\mathrm{Al}(111)$ plane] on freshly cleaned $n$-type $\operatorname{Si}(111)-7 \times 7$ surfaces followed by an anneal at $970 \mathrm{~K} .{ }^{14}$ The following Al film growth was performed on these substrates at $110 \mathrm{~K}$. After growth, the deposited film was warmed up to room temperature and investigated in situ by scanning tunneling microscopy (STM) and low energy electron diffraction (LEED) in ultrahigh vacuum $\left(p<10^{-8} \mathrm{~Pa}\right)$.

Figure 1 shows an overview of the $\mathrm{Al}$ growth on $\mathrm{Si}(111)-\sqrt{3} \times \sqrt{3}-\mathrm{Al}$ substrates. In the initial stages, a well ordered, atomically smooth, and complete wetting layer is formed after deposition of $0.82 \pm 0.1 \mathrm{ML} \mathrm{Al}$ [Fig. 1(a)]. The visible steps with a height of $3.1 \AA$ arise from steps present on the Si substrate before growth [Fig. 1(a2)]. The LEED pattern (inset) shows sharp sixfold $1 \times 1$ spots corresponding to the lattice constant of a close-packed $\mathrm{Al}(111)-1 \times 1$ threefold plane. LEED spots corresponding to $\mathrm{Si}(111)-1 \times 1$ are also observed, while the spots of the $\sqrt{3} \times \sqrt{3}$ reconstruction disappeared. This suggests that upon the formation of the wetting layer, the $\sqrt{3} \times \sqrt{3}$ substrate is converted to a $\mathrm{Si}(111)-1 \times 1-\mathrm{Al}(111)-1 \times 1$ interface. During this process, the $\mathrm{Al}$ adatoms of the initial $\sqrt{3} \times \sqrt{3}$ reconstruction are incorporated into the $1 \times 1 \mathrm{Al}$ wetting layer. This is corroborated by the observation that $0.82 \pm 0.1 \mathrm{ML}$ instead of $1 \mathrm{ML}$ $\mathrm{Al}$ is sufficient for forming a complete $1 \times 1 \mathrm{Al}$ wetting layer. The missing $0.18 \pm 0.1 \mathrm{ML}$ is provided by the $0.15 \mathrm{ML} \mathrm{Al}$ adatoms of the $\sqrt{3} \times \sqrt{3}$ reconstruction. Thus, in the initial 


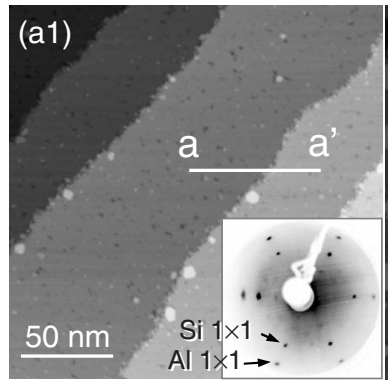

(a2)

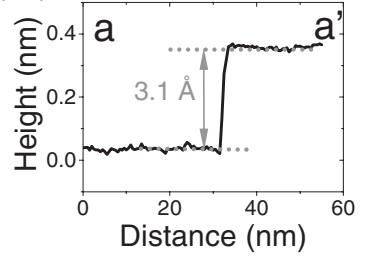

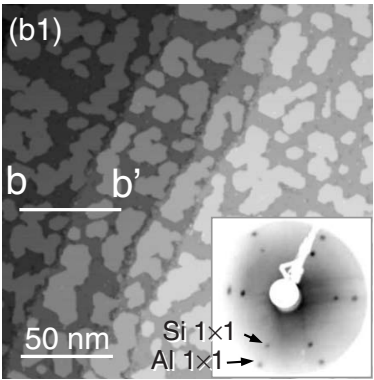

(b2)

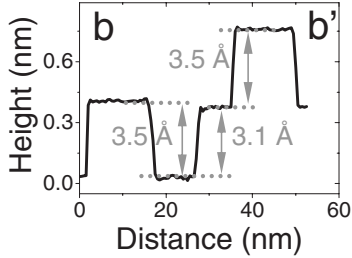

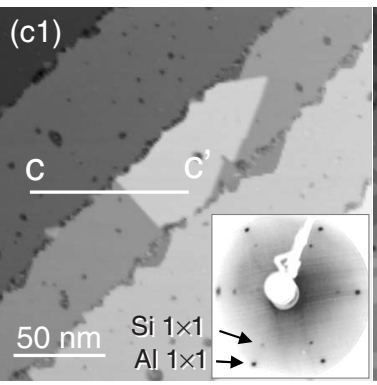

(c2)

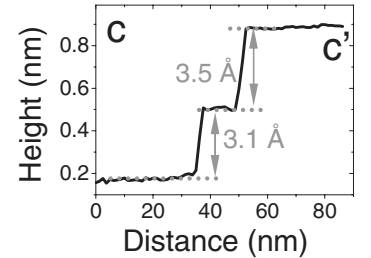

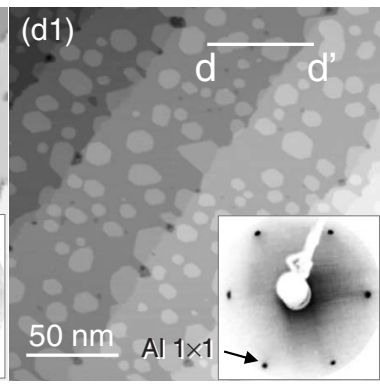

$(\mathrm{d} 2)$

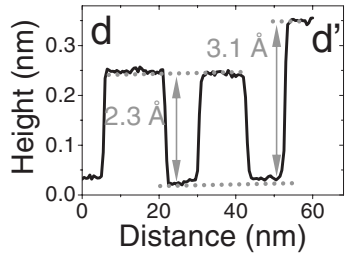

FIG. 1. Overview of the growth of the first few layers of $\mathrm{Al}$ on $\mathrm{Si}(111)-\sqrt{3} \times \sqrt{3}-\mathrm{Al}$ substrates. (a1) STM images of a completed $1 \times 1 \mathrm{Al}$ wetting layer with its LEED pattern as inset. No $\sqrt{3} \times \sqrt{3}$ spots remain. (a2) The steps in (a1) have the height of the steps on the Si substrate before growth. (b1) $3.5 \AA$ high Al islands [see (b2) for height] grow two dimensionally on the wetting layer. Inset: Corresponding LEED pattern. (c1) A completed $3.5 \AA$ thick Al film is formed, on which a second-layer (triangular) island nucleates, whose height is again $3.5 \AA$ [see (c2)]. Inset: Corresponding LEED pattern, which shows sharp $1 \times 1$ spots, indicates that the top layer is a $1 \times 1$ ordered $\mathrm{Al}(111)$ layer. (d1) After the second $3.5 \AA$ thick Al layer is completed, the growth switches to the nucleation of islands with a normal $\mathrm{Al}(111)-1 \times 1$ height of $2.3 \AA$ [see (d2)]. The corresponding LEED pattern is shown in the inset. [(a2)-(d2)] Corresponding height profiles illustrating the different thicknesses of the islands in (a1)-(d1). Note that the $3.1 \AA$ high steps arise from steps on the Si substrate.

growth stage, a $1 \times 1 \mathrm{Al}$ wetting layer is formed on top of a Si(111)- $1 \times 1$ surface.

The most striking finding is, however, that further deposition of $\mathrm{Al}$ on the wetting layer leads to $3.5 \AA$ high islands corresponding to $\approx 1.5 \mathrm{ML}$ [see STM image in Fig. 1(b1)]. These islands eventually form a perfectly smooth $3.5 \AA$ thick film. The unusual $3.5 \AA$ height of the islands is clearly visible in the height profiles shown in Fig. 1(b2). The LEED pattern (see inset) indicates the presence of an $\mathrm{Al}(111)$ $1 \times 1$ layer terminating the $3.5 \AA$ thick film.

On top of this film, apparently yet another layer of $3.5 \AA$ high islands forms with further Al deposition [Fig. 1(c1) and corresponding height profile Fig. 1(c2)]. The LEED pattern again indicates an $\mathrm{Al}(111)-1 \times 1$ top layer (inset). Further growth proceeds in a layer-by-layer mode with step heights of $2.3 \AA$, corresponding to the normal interlayer spacing between neighboring close-packed $\mathrm{Al}(111)$ planes, i.e., $1 \mathrm{ML}$ of $\mathrm{Al}$ [see Fig. 1(d), LEED pattern and height profile]. The island heights did not change in a detectable manner $(<0.2 \AA)$ within a voltage range of $\pm 5 \mathrm{~V}$ applied to the sample, indicating that electronic effects do not influence the height measurements.

In order to understand the unusual step heights and the stacking of the Al film, we carefully measured the coverage of successive layers as a function of the amount of $\mathrm{Al}$ deposited. Figure 2 demonstrates that the first two $3.5 \AA$ thick $\mathrm{Al}$ layers require $1.46 \pm 0.12$ and $1.55 \pm 0.13 \mathrm{ML}$ for completion, respectively, whereas the following $2.3 \AA$ thick layers require only close to $1 \mathrm{ML}$. Furthermore, all LEED patterns always indicate that the top terminating layer is a closepacked $\mathrm{Al}(111)-1 \times 1$ plane (see, e.g., Fig. 1). Thus, the coverage information in Fig. 2 combined with the LEED information indicates that the first $3.5 \AA$ thick Al layer consists of a stack of two combined planes of $\mathrm{Al}$ atoms: the top layer being a dense close-packed $1 \times 1$ plane $(1 \mathrm{ML})$ and the underlying layer being a loosely packed dilute layer containing only $0.46 \pm 0.12 \mathrm{ML} \mathrm{Al}$ atoms. The existence of a dilute layer below the dense close-packed layer can be further corroborated by analyzing the moiré pattern visible on top of the first $3.5 \AA$ thick Al layer [Fig. 3(a)]. The Fourier transform of the moiré pattern [Fig. 3(b)] shows sixfold spots pointing to a

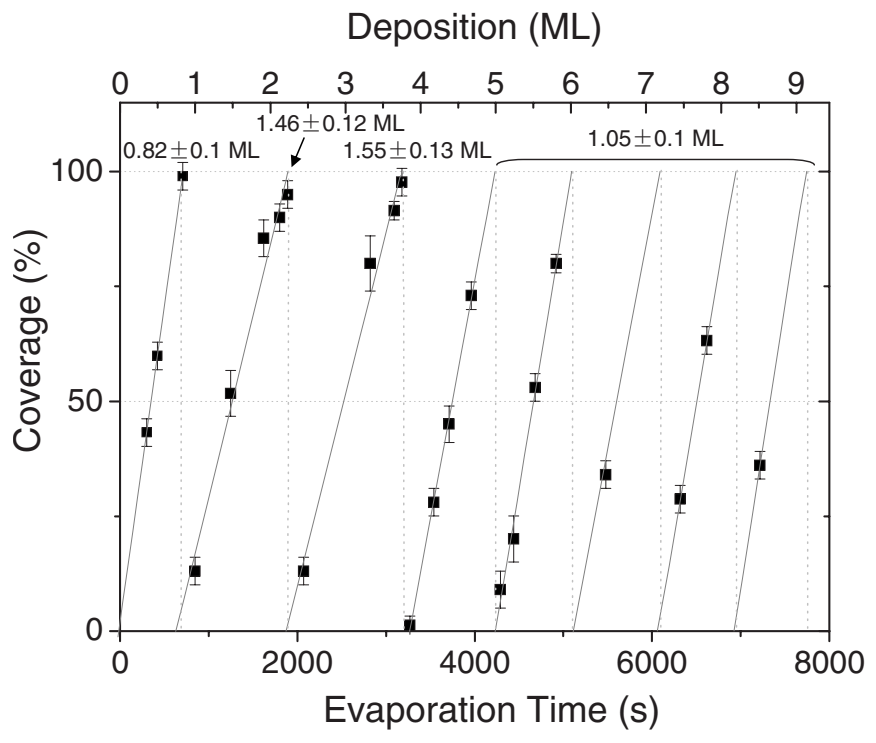

FIG. 2. Coverage area of successive layers as a function of the amount of $\mathrm{Al}$ deposited. The wetting layer consumes $0.82 \pm 0.1 \mathrm{ML}$ Al. The first two $3.5 \AA$ thick Al layers on top of the wetting layer each require around 1.5 ML of $\mathrm{Al}$ atoms. The following $2.3 \AA$ thick layers necessitate only close to $1 \mathrm{ML}$ of $\mathrm{Al}$ per layer. 

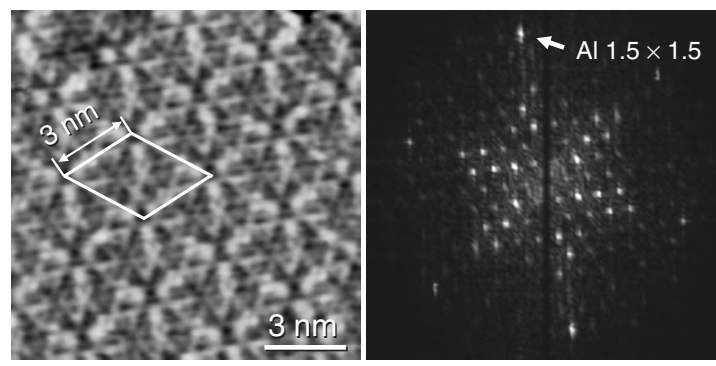

(a)

(b)

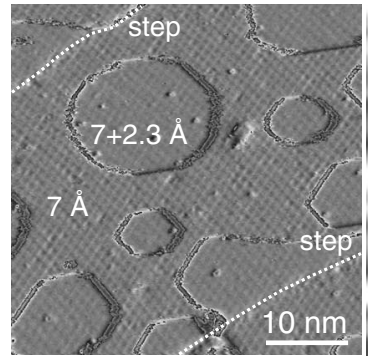

(c)

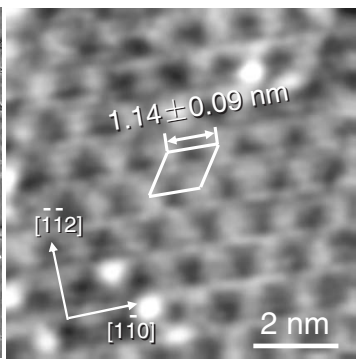

(d)

FIG. 3. (a) Moiré pattern on top of the first $3.5 \AA$ thick film grown on the wetting layer. (b) Fourier spectrum of (a) illustrating the $\mathrm{Al} 1.5 \times 1.5$ periodicity yielding a moiré pattern periodicity of $10.5 a$, with $a$ being the $\operatorname{Al}(111) 1 \times 1$ lattice constant. (c) Moiré pattern on top of the 7 and $7+2.3 \AA$ films grown on the wetting layer. Note the similarity of the two moiré patterns. The indicated steps are substrate steps. (d) High-resolution STM image of the moiré pattern on the $\mathrm{WL}+7+2.3 \AA$ islands.

threefold $1.5 \times 1.5$ layer below the $\mathrm{Al}(111) 1 \times 1$ plane. $\mathrm{A}$ detailed analysis of the Fourier spectrum indicates that the $1.5 \times 1.5$ layer is rotated by about $8^{\circ}$ relative to the overlying $\mathrm{Al}(111) 1 \times 1$ plane. This results in a moiré pattern with a lattice constant of $10.5 a \approx 3.0 \mathrm{~nm}$ [ $a$ being the lattice constant in the $\mathrm{Al}(111) 1 \times 1$ plane], in good agreement with the STM image in Fig. 3(a). A further support of the existence of the dilute $1.5 \times 1.5$ layer is its theoretical density of $0.44 \mathrm{ML}$, which is consistent with the measured density of $0.46 \pm 0.12$ ML in the dilute layer. We, therefore, infer that the underlying layer has a dilute $1.5 \times 1.5 \mathrm{Al}$ structure and is well ordered.

The presence of dilute atomic layers can be further corroborated by analyzing STM images measured right after the preparation of the films, when the films have not been fully warmed up yet. Films in such a state exhibit a varying density of small islands with smaller heights [see, e.g., arrows in Fig. 4(a)]. On the wetting layer, we observed in addition to the $3.5 \AA$ high islands the nucleation of $1.4 \AA$ high islands, as shown in the height histogram in Fig. 4(c). These small islands are thermally unstable and form upon warm-up $3.5 \AA$ high islands. Figure 4(b) shows an atomically resolved image of a small $1.4 \AA$ high island area, showing a partially ordered arrangement of atoms. In the better ordered regions, the separations between the maxima are still larger, but getting close to those expected in a $1.5 \times 1.5 \mathrm{Al}$ layer. On top of the first $3.5 \AA$ thick layer, a number of islands with smaller heights also nucleate [Figs. 4(d) and 4(e)]. We observed primarily small islands with a height of $1.4 \AA$, consistent with a

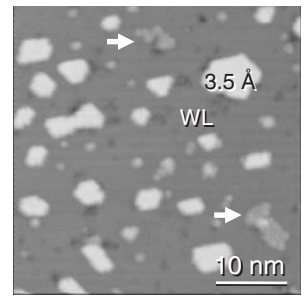

(a)

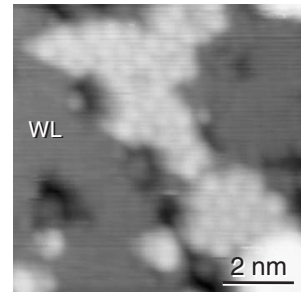

(b)

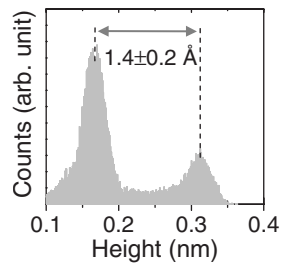

(c)

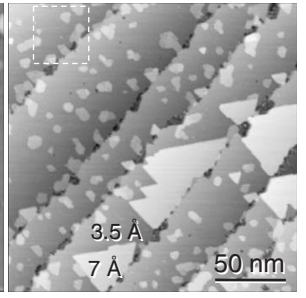

(d)

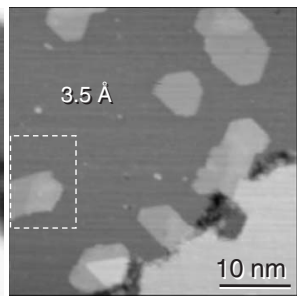

(e)

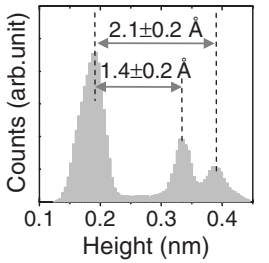

(f)
FIG. 4. STM images of Al films obtained at low temperature before complete warm-up. (a) Initial growth stages of the growth of the first $3.5 \AA$ high layer. In addition to $3.5 \AA$ high islands, small $1.4 \AA$ islands exist (arrows). (b) High-resolution STM image of such an island, showing a partially ordered structure. Note the nondense atomic arrangement. (c) The histogram of the distribution of heights obtained from (b) shows that islands are $1.4 \pm 0.2 \AA$ high. (d) Initial stages of growth of the second $3.5 \AA$ high layer. Again, small islands with heights lower than $3.5 \AA$ can be observed. (e) The zoom-in image of the marked area in (d). Two different heights can be observed. (f) The histogram of the distribution of heights obtained from the area marked in (e) illustrates the presence of 1.4 and $2.1 \AA$ islands.

dilute layer, and less frequently of $2.1 \AA$, consistent with a $1 \times 1$ Al layer [see height frequency distributions in Fig. 4(f)]. These observations illustrate that in the investigated system, dilute Al layers indeed exist, supporting the above derived bilayer model of the $3.5 \AA$ thick Al layers.

At this stage, we address the number of periods of dilutedense layer stacks, which can be grown. The height of the terraces and the atom densities suggest that two periods of dilute-dense layer stacks grow. However, if we analyze the moiré pattern on top of the second $3.5 \AA$ thick Al layer and on further successive monolayers, we obtain a somewhat different picture. Figure 3(c) shows that the moiré patterns on top of the second $3.5 \AA$ thick Al layer (labeled $7 \AA$ ) and on top of a further grown monolayer (labeled $7+2.3 \AA$ ) are essentially identical. Figure 3(d) illustrates that the moiré pattern has a lattice constant of $1.14 \pm 0.09 \mathrm{~nm}$ and is oriented parallel to the $\langle 110\rangle$ directions of the substrate. There are no indications of any weak leftover moiré pattern of the first $3.5 \AA$ layer. These orientation and dimension values arise 


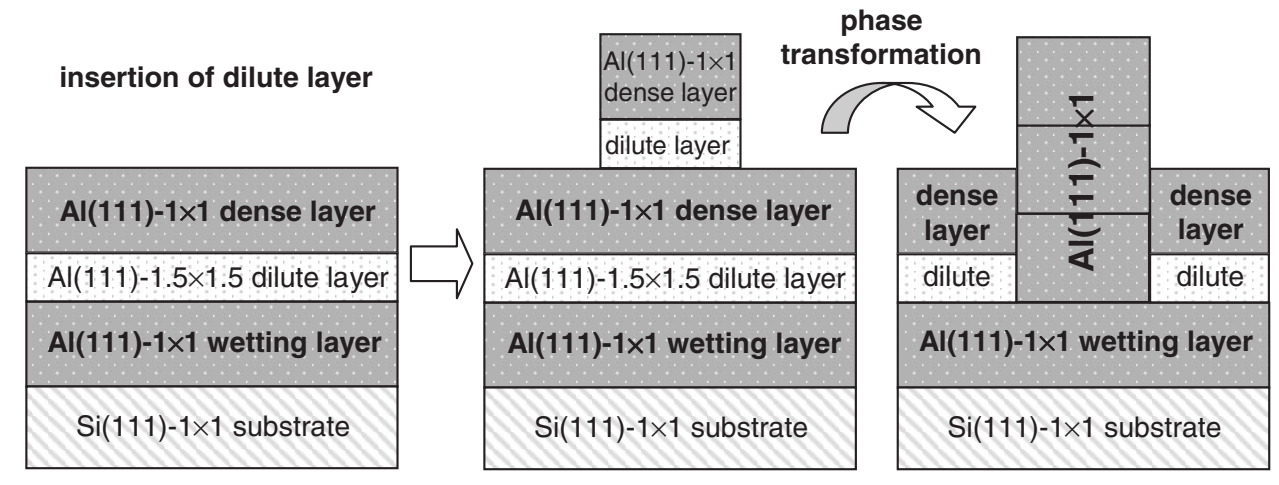

FIG. 5. Schematic drawing of the growth mode of QSEinduced insertion of dilute layers and a strain-driven phase transformation. from the mismatch of the $\mathrm{Si}(111)-1 \times 1$ lattice with a closepacked $\mathrm{Al}(111)-1 \times 1$ lattice. The 3:4 Al:Si lattice constant ratio yields without rotation a moiré pattern having a lattice constant of $1.144 \mathrm{~nm}$. This is in excellent agreement with the observations. Thus, the moiré pattern indicates that the whole film, down to the substrate, transformed from alternating dense-dilute layers into dense fcc stacking of $\mathrm{Al}(111)-1 \times 1$ layers once the second $3.5 \AA$ thick film forms. This is further corroborated by the density measurement in Fig. 2, which shows that a fully completed $7 \AA$ thick film contains almost exactly $3 \mathrm{ML}$ Al $(1.46+1.55 \mathrm{ML})$. From this, we can conclude that the dense-dilute layers grow up to two periods (wetting layers, first dilute layer, dense layer, and metastable nucleation of dilute islands) at low temperatures. However, at room temperature, only one period, i.e., the dense wetting layer, the dilute layer, and the terminating dense layer, is stable. Beyond this, further deposition leads to the phase transformation. Note that unless the $7 \AA$ thick film is fully completed, the two phases, i.e., the alternation of dense and dilute layers and the close-packed fcc structure, will coexist (due to the copresence of 3.5 and $7 \AA$ thick films). The observed growth mode is schematically summarized in Fig. 5.

At this point, we probed the relative stability of the different film thicknesses by annealing a $2.4 \mathrm{ML}$ thick Al film $30 \mathrm{~h}$ at room temperature. During this process, the film gradually breaks into islands with various heights on top of the wetting layer [Fig. 6(a)]. We measured the resulting distribution of the area occupation $P(d)$ of films with different thicknesses $d$ [inset in Fig. 6(a)]. We observed film thicknesses on top of the wetting layer of 3.5 and $7 \AA$ as well as of $(7+n \times 2.3) \AA$, with $n \in\{1,2,3,4\}$ [corresponding to one to four layers of $1 \times 1 \mathrm{Al}(111)$ on top of the $7 \AA$ high islands]. However, 2.3 and $4.6 \AA$ high islands, corresponding to one and two layers of $1 \times 1 \mathrm{Al}(111)$, are not observed.

This distribution does not change anymore after $20 \mathrm{~h}$ annealing, indicating that the film reached its so-called local equilibrium, i.e., the metastable $\mathrm{Al}$ film reached a local equilibrium defined by the constraint that the Si-Al interface remained unaltered and sharp and that the barrier for the chemical reaction leading to the formation of aluminum silicides is not overcome. This situation requires an analysis under so-called local equilibrium conditions, as outlined in Ref. 15: The variations in the distribution of the area occupation are indicative for the relative preference of certain film thicknesses over others. Experimentally, the relative stability of islands can be estimated by comparing the fractional

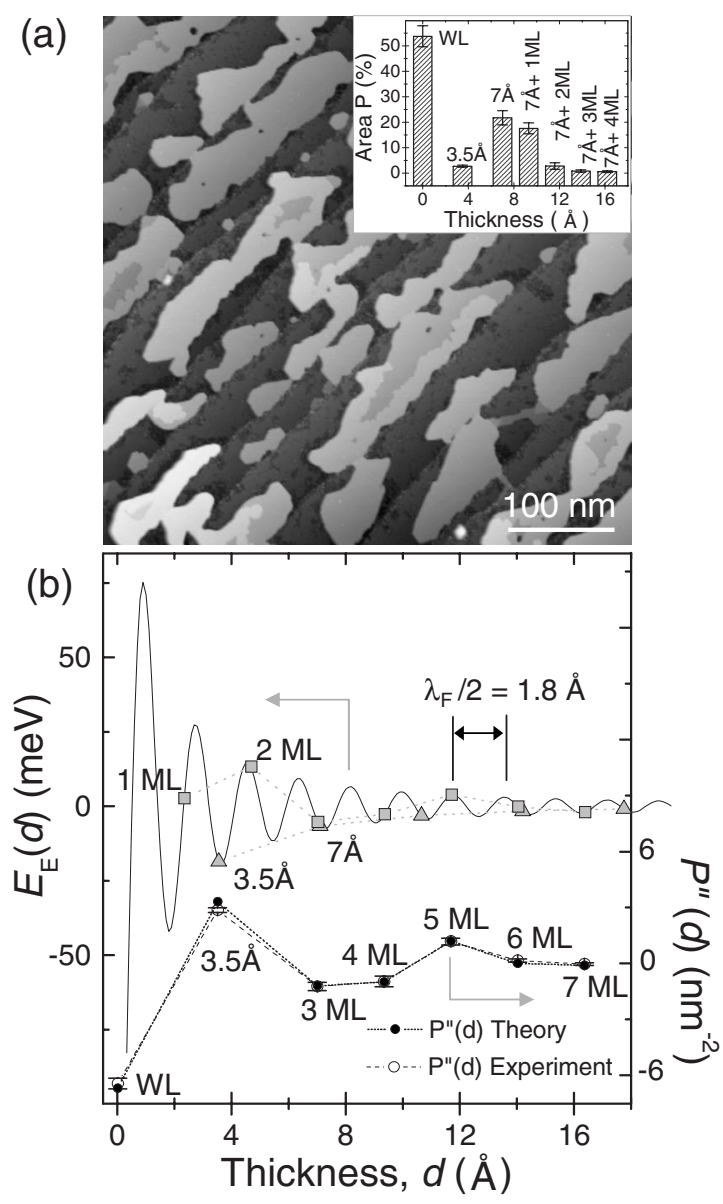

FIG. 6. (a) Example of an STM image of a 2.4 ML thick film equilibrated by annealing for $30 \mathrm{~h}$ at room temperature. From such images, the coverage area distribution $P(d)$ of the different film thicknesses $d$ was determined (see inset). (b) Derivation of the electron energy per $\mathrm{Al}$ atom as a function of the film thickness (solid line, left axis): As a measure of the film stability, the discrete second derivative of the $P(d)$ is extracted (open circles, right energy scale) and modeled using a quantum size effect governed electron energy curve in the form of a Friedel oscillation. The best fit of the $P^{\prime \prime}(d)$, shown as filled circles, nicely agrees with the experimental data (see text for method and fitting parameters). The solid line shows the oscillating electron energy curve per atom $E_{E}(d)$ obtained from the fit. The two sets of gray symbols illustrate the sampling of the surface energy for a growth mode with normal stacking of $\mathrm{Al}(111)$ planes (squares) and with alternating dense and dilute layers (triangles). 
surface area a certain film thickness occupies with that of its neighboring thicknesses, with which it is in equilibrium. This can be done using the discrete second derivative of $P(d)$, $P^{\prime \prime}(d)=\partial^{2} P(d) / \partial d^{2} .{ }^{15}$ Film thicknesses having $P^{\prime \prime}(d)<0$ are relatively stable, while thicknesses with $P^{\prime \prime}(d)>0$ are unstable. Applying this principle to our case, we have first to address with which thicknesses a particular layer is in equilibrium. For large film thicknesses, the film grows in a normal layer-by-layer mode. Thus, in this case, the $n$th monolayer is in equilibrium with the monolayers $n+1$ and $n-1$. At small thicknesses, the first $3.5 \AA$ thick layer is of particular interest. At first sight, the neighboring film thicknesses, with which the first $3.5 \AA$ thick layer should be in equilibrium, may appear to be the 1 and 2 ML thick layers. These are, however, not observed. Instead, time dependent measurements show that the first $3.5 \AA$ thick layer is rather in equilibrium with the wetting layer and the $7 \AA$ thick layer on top of the wetting layer. Thus, the value $P^{\prime \prime}(d)$ has to be calculated taking only the wetting, the $3.5 \AA$, the $7 \AA$, and all following monolayers into account. We numerically obtained the experimental second derivative using $P^{\prime \prime}\left(d_{i}\right)$ $=\left[P\left(d_{i-1}\right)-P\left(d_{i}\right)\right] /\left[\left(d_{i-1}-d_{i}\right)\left(d_{i-1}-d_{i+1}\right)\right]-\left[P\left(d_{i}\right)-P\left(d_{i+1}\right)\right] /$ $\left[\left(d_{i}-d_{i+1}\right)\left(d_{i-1}-d_{i+1}\right)\right]$, with $d_{i}$ being the thickness of the film with $i$ layers grown. The results of $P^{\prime \prime}$ thus obtained are identical to those obtained by using the curvature of a parabola fit. The such obtained experimental $P^{\prime \prime}(d)$ values are shown as open circles in the bottom of Fig. 6(b) (right axis).

In order to understand the experimental values of relative stability, we have to recall that the film's total energy is composed of the energy of the atomic arrangement (strain) and the energy of the electron system (electron energy). At first, one may think that only strain induces the dilute-dense structure. However, the almost "magic lattice match" between $\mathrm{Si}$ and $\mathrm{Al}\left(3 \times a_{\mathrm{Si}} \approx 4 \times a_{\mathrm{Al}}\right)$ and the fact that the introduction of strain increases the energy as compared to the unstrained configuration rule out strain as only driving force. The electron energy can, however, lower the film energy. Thus, we have to consider the combined action of both strain and electron energy. Based on these concepts, we modeled the energy of the film using an electron energy $E_{E}(d)$ governed by the quantum size effect as outlined below and a strain energy $E_{S}(d)$. For the strain energy, we only considered strain in the dilute-dense layer stack but not in the normal grown monolayers. The normal grown monolayers can be expected to have only very small changes in their strain energy as compared to unstrained bulk fcc $\mathrm{Al}$ because of the almost magic lattice match between $\mathrm{Si}$ and $\mathrm{Al}$.

We now address the electron energy. The observed layer thickness of $3.5 \AA$ coincides with the Fermi wavelength $\lambda_{F}$ of $\mathrm{Al}$ of $3.6 \AA,{ }^{16,17}$ but not with any length scale of the $\mathrm{Al}$ crystal structure. This indicates that electronic effects, namely, the QSE arising from the confinement of the electrons in the film, play a role. We recall that the QSE induces oscillations of electron energy of thin films with thicknesses $d$ comparable to the Fermi wavelength $\lambda_{F}{ }^{1,2}$ The modulation of the average electron energy per atom as a function of the film thickness $d$ is given by ${ }^{2,15} E_{E}(d)=A \sin \left[2 k_{F}(d+\phi)\right] /(d$ $+\phi)^{\alpha}+B$, with $k_{F}=2 \pi / \lambda_{F}$ being the Fermi wave vector, $\phi$ the phase shift arising at the film boundaries, and $\alpha$ the damping coefficient. $A$ and $B$ are constants. The equation describes a damped Friedel oscillation with a wavelength of $\lambda_{F} / 2$.

In order to compare this model (electron energy plus strain energy) with our $\mathrm{Al}$ thin film, we calculate the discrete second derivative of the theoretical occupation area distribution $P_{\text {theo }}(d)$, following the methodology of Ref. 15. Note that, however, in Ref. 15, identical layer thicknesses were always assumed, which is not the case in our system. Therefore, we adapted the methodology of Ref. 15 to different layer thicknesses by normalizing the formulas by the differences in film thicknesses $\left(d_{i}-d_{i+1}\right)$ between neighboring films with $i$ and $i+1$ layers. Furthermore, the distribution function used in Ref. 15 assumes constant atom densities and uses only the surface electronic energy instead of the total energy. This is also not applicable to our system and thus we modified the distribution function $P_{\text {theo }}(d)$ $=C \exp \left[-E_{\text {tot }}(d) \rho(d) / k_{B} T\right]$ with $\rho(d)$, which is the vertical density of $\mathrm{Al}$ atoms, i.e., the number of atoms in one column of a film of thickness $d$, and $E_{\text {tot }}(d)$, which is the total energy per $\mathrm{Al}$ atom. The total energy is approximated by the sum of the electronic and strain energy by using $E_{\mathrm{tot}}(d)=E_{E}(d)$ $+E_{S}(d)$. We only included a strain of $E_{S}$ for the $3.5 \AA$ thick layer by using $E_{S}(d)=E_{S} \delta(d-3.5 \AA)$. For all other film thicknesses, the strain was approximated by zero.

If we apply this model to our data, we first fit the data at larger thicknesses with normal layer-by-layer growth, because there strain effect can be essentially neglected. In this case, we have in principle three fitting parameters $(A, \alpha$, and $\phi)$ to be adjusted to provide a best fit to $P^{\prime \prime}(d)$. However, the parameters $A$ and $\alpha$ exhibit a significant covariance: ${ }^{15}$ Within a very large range of $A$ and $\alpha$ pairs, the energy curve is almost unchanged at large film thicknesses. Unfortunately, at small film thicknesses, this leads to largely deviating energy values. Note that the wavelength is not affected by this and thus the physical interpretation given below remains unaffected. Nevertheless, in order to avoid this uncertainty, we calculated $\alpha$ as outlined in Ref. 15 using the free electron model and as parameters the atom density, the number of free electron per Al atom (3), and the Fermi wave vector. We obtained an $\alpha$ of 1.68. The resulting best fit yields $A$ $=287 \pm 50 \mathrm{meV}$ and $\phi=0.1282 \pm 0.002 \mathrm{~nm}$ and the corresponding electron energy [damped Friedel oscillation $E_{E}(d)$ ] curve per atom is shown as solid line in Fig. 6(b) (left axis). For the best fit of all the $P^{\prime \prime}(d)$ values we then included a strain energy of $37 \pm 20 \mathrm{meV} /$ atom for the $3.5 \AA$ thick film only. The resulting theoretical $P^{\prime \prime}(d)$ values are shown as filled circles in the bottom of Fig. 6(b) (right axis). They agree well with the experimental values (open circles). Without strain, the agreement is very poor at small thicknesses.

The derived electron energy curve [solid line in Fig. 6(b)] allows us to understand the essential physics governing the growth mode. First, we focus on the insertion of dilute atomic layers between dense layers. The two different growth modes, i.e., the normal stacking of $\mathrm{Al}(111)-1 \times 1$ and the observed alternating dense and dilute layers, sample different film thicknesses along the energy curve highlighted by gray squares and triangles in Fig. 6(b), respectively. The comparison of both demonstrates that the observed growth 
mode (dilute and/or dense layers) has a significantly lower electron energy than a normal stacking of dense $\mathrm{Al}(111)$ $1 \times 1$ planes for thicknesses below $7 \AA$. Thus, the electron energy prefers to form initially $3.5 \AA$ thick layers, consistent with our observations.

The electron energy does, however, not include the additional energy required for the insertion of dilute layers. Our estimation of the strain energy per atom for the $3.5 \AA$ thick film suggests that most of the gain in electron energy per atom is consumed by the strain energy per atom. However, the fact that the $3.5 \AA$ film is stable as compared to the 1 and 2 ML films shows that the sum of strain and electron energy is still lower than for dense 1 and 2 ML films. Several reasons support this. Firstly, the accuracies of the strain energy estimate and of the electron energy curve at small thicknesses (recall $A$ and $\alpha$ covariance affecting strongly the energy at small $d$ ) are limited and may well provide within the error margins a clear energy gain. Secondly, the atom density in the dilute layer reduces the number of atoms in the film and thus at constant energy per atom, the film energy of the dilute and/or dense stacking is lowered. Thirdly, the reduction of the atoms in the dilute layers reduces the number of strained bonds significantly, thereby reducing the strain energy of the whole film. Thus, one can conclude that at small thicknesses, the insertion of dilute layers is driven by the gain in electron energy governed by the QSE.

The strain energy becomes, however, decisive for vanishing energy differences in the electron energy between the two growth modes at larger thicknesses. For example, films on top of the wetting layer with $7 \AA$ thickness have almost the same electron energy in both phases, the alternating dilute and/or dense stacking and $3 \mathrm{ML}$ of dense $\mathrm{Al}(111)$ $1 \times 1$ planes. In such circumstances, the strain energy arising from the insertion of dilute layers prefers the close-packed stacking over the alternating dilute and/or dense stacking and one can expect the film to undergo a phase transformation to relax the strain. This agrees well with the experimentally indicated phase transformation for $7 \AA$ thick films. Further growth will then proceed in a normal layer-by-layer stacking of dense $\mathrm{Al}(111)-1 \times 1$ planes, despite that the alternating dilute and/or dense stacking would still have a lower electron energy (about $10 \mathrm{meV}$ at $d=10.5 \AA$ ).

Finally, the influence the Al-Si interface on the growth of
Al can be discussed best by comparing our system with the growth of $\mathrm{Al}$ on $\mathrm{Si}(111)-7 \times 7$ substrates. In contrast to our system, the $\mathrm{Al}$ film grown on $\mathrm{Si}(111)-7 \times 7$ substrates forms only above $4 \mathrm{ML}$ fully covering and wetting overlayers within a normal stacking of $\mathrm{Al}(111)-1 \times 1$ planes. ${ }^{18}$ Below 4 ML, the film breaks up into islands, whose exact height is unclear. In our system even below 4 ML thickness, the film forms fully wetting layers. This suggests that the driving force for stabilization (i.e., QSE) of ultrathin films below 4 ML is weaker for $\mathrm{Al}$ growth on $\mathrm{Si}(111)-7 \times 7$ than on $\mathrm{Si}(111)-\sqrt{3} \times \sqrt{3}$ substrates. Since the only difference of this system and ours is the interface structure, the lack of QSE in the case of the Al films on $\mathrm{Si}(111)-7 \times 7$ surfaces is likely correlated to the poorly defined interface characterized by a disordered $\mathrm{Al}$ wetting layer on top of a partially preserved $7 \times 7$ reconstruction, while in our case, a perfectly sharp $\mathrm{Al} / \mathrm{Si}$ interface is found.

In conclusion, we demonstrated using STM, LEED, and coverage measurements that $\mathrm{Al}$ thin films on $\mathrm{Si}(111)$ $\sqrt{3} \times \sqrt{3}$ - Al substrates form film structures consisting of inserted dilute $1.5 \times 1.5$ atomic layers for small thicknesses ( $<$ wetting layer $+7 \AA$ ). This structure allows the $\mathrm{Al}$ film to reach thicknesses perfectly well adjusted to the minima of the oscillating electron energy, which arises from the spatial confinement of the free electrons in the thin film. Such thicknesses cannot be achieved by contractions or expansions only of normally stacked dense $\mathrm{Al}(111)-1 \times 1$ layers due to the excessive strain needed. Thus, the film's structure deviates at small thicknesses from the close-packed principle of metals due to quantum size effects. At larger thicknesses (> wetting layer $+7 \AA$ ), where the differences in electron energy become small, strain induces a phase transformation from the dilute and/or dense stacking to a normal stacking of $\mathrm{Al}(111)-1 \times 1$ layers, thereby driving the film back to a close-packed atomic structure. This example highlights that the quantum size effect affects not only a number of physical properties of thin films but also modifies the atomic structure itself and the atomic density in low dimensional systems.

The authors thank Junren Shi for valuable discussions on the model of island stability. The work was supported by the National Natural Science Foundation (No. 60021403), MOST, and CAS of China and partially by the Deutsche Forschungsgemeinschaft.
${ }^{1}$ F. K. Schulte, Surf. Sci. 55, 427 (1976).

${ }^{2}$ Z. Zhang, Q. Niu, and C. K. Shih, Phys. Rev. Lett. 80, 5381 (1998); J. H. Cho, Q. Niu, and Z. Y. Zhang, ibid. 80, 3582 (1998).

${ }^{3}$ J. J. Paggel, T. Miller, and T. C. Chiang, Science 283, 1709 (1999).

${ }^{4}$ T. C. Chiang, Surf. Sci. Rep. 39, 181 (2000) and references therein.

${ }^{5}$ K. Budde, E. Abram, V. Yeh, and M. C. Tringides, Phys. Rev. B 61, R10602 (2000); R. Otero, A. L. Vasquez de Parga, and R. Miranda, ibid. 66, 115401 (2002); V. Yeh, L. Berbil-Bautista, C.
Z. Wang, K. M. Ho, and M. C. Tringides, Phys. Rev. Lett. 85, 5158 (2000).

${ }^{6}$ A. Crottini, D. Cvetko, L. Floreano, R. Gotter, A. Morgante, and F. Tommasini, Phys. Rev. Lett. 79, 1527 (1997).

${ }^{7}$ W. B. Su, S. H. Chang, W. B. Jian, C. S. Chang, L. J. Chen, and T. T. Tsong, Phys. Rev. Lett. 86, 5116 (2001).

${ }^{8}$ P. Czoschke, H. W. Hong, L. Basile, and T. C. Chiang, Phys. Rev. Lett. 91, 226801 (2003).

${ }^{9}$ Y. Guo et al., Science 306, 1915 (2004).

${ }^{10}$ D. A. Ricci, T. Miller, and T. C. Chiang, Phys. Rev. Lett. 95, 266101 (2005). 
${ }^{11}$ C. A. Jeffrey, E. H. Conrad, R. Feng, M. Hupalo, C. Kim, P. J. Ryan, P. F. Miceli, and M. C. Tringides, Phys. Rev. Lett. 96, 106105 (2006).

${ }^{12}$ K. J. Chao, Z. Y. Zhang, P. Ebert, and C. K. Shih, Phys. Rev. B 60, 4988 (1999).

${ }^{13}$ C. S. Jiang, H. B. Yu, C. K. Shih, and P. Ebert, Surf. Sci. 518, 63 (2000).

${ }^{14}$ R. J. Hamers, Phys. Rev. B 40, 1657 (1989).

${ }^{15}$ P. Czoschke, H. W. Hong, L. Basile, and T. C. Chiang, Phys. Rev. Lett. 93, 036103 (2004); Phys. Rev. B 72, 075402 (2005).

${ }^{16}$ P. J. Feibelman, Phys. Rev. B 27, 1991 (1983); P. J. Feibelman and D. R. Hamann, ibid. 29, 6463 (1984).
${ }^{17}$ In a jellium model, the Fermi wavelength $\lambda_{F}$ of our Al superlattice can be estimated using $k_{F}=\left(3 \pi^{2} n\right)^{1 / 3}$ and $k_{F}=2 \pi / \lambda_{F}$, with $n$ being the density of free electrons. For our $0.35 \mathrm{~nm}$ thick film containing 1.4-1.5 ML of $\mathrm{Al}$ atoms, this yields $\lambda_{F(\text { film })}$ $=(0.974-0.996) \times \lambda_{F(\mathrm{Al})}$, with $\lambda_{F(\mathrm{Al})}=0.36 \mathrm{~nm}$. Since the jellium model was, however, shown to underestimate $\lambda_{F}$ by $\approx 5 \%$ as compared to a self-consistent LCAO calculation [P. J. Feibelman, Phys. Rev. B 27, 1991 (1983)], we used as first approximation $\lambda_{F(\text { film })}=\lambda_{F(\mathrm{Al})}=0.36 \mathrm{~nm}$.

${ }^{18}$ H. Liu, Y. F. Zhang, D. Y. Wang, M. H. Pan, J. F. Jia, and Q. K. Xue, Surf. Sci. 571, 5 (2004). 